\title{
Hipertensión portal secundario a trombosis de la vena porta y várices esofágicas
}

Isabel Ruiz Ponce de león ${ }^{\text {a, }}$, Esteban Rodríguez-Cárdenas ${ }^{\text {a,b }}$, Víctor Hugo Villarreal Cortes ${ }^{\mathrm{c}}$,

Rodrigo Guarín Rojas ${ }^{\mathrm{d}}$, José Fernando Gómez Urrego ${ }^{\mathrm{e}, \mathrm{b}}$.

\begin{abstract}
${ }^{a}$ Médica residente de Pediatría Universidad Libre seccional Cali, ORCID: https://orcid.org/0000-0002-4018-003X, Isaruizp201192@gmail.com
${ }^{\mathrm{b}}$ Médico Pediatra, Grupo Investigación en Pediatría GRINPED 0142019. ORCID: https://orcid.org/0000-0003-2892-2317, Estebanroca17@hotmail.com ; c Médico Pediatra- Fundación Clínica Infantil Club Noel, Docente posgrado de Pediatría de la Universidad Libre, seccional Cali. ORCID: https://orcid.org/00000003-0329-510X , Victorh.villarrealc@unilibre.edu.co ; ${ }^{\mathrm{d}}$ Médico interno, Universidad Libre seccional Cali ORCID: https://orcid.org/0000-0002-4416-6139, Roguja8@hotmail.com ; ${ }^{\mathrm{e}}$ Médico Pediatra- Fundación Clínica Infantil Club Noel, Coordinador posgrado de Pediatría de la Universidad Libre seccional Cali, Epidemiólogo. ORCID: https://orcid.org/0000-0003-4708-7759, postgradospedul@gmail.com .
\end{abstract} DOI: https://doi.org/10.22517/25395203.23981

\section{Resumen}

La hipertensión portal se define como la alteración patológica en el gradiente de presión a nivel del sistema portal, es decir, la diferencia entre la presión de la vena porta y la vena cava inferior. El valor normal es entre 1-5 $\mathrm{mm} \mathrm{Hg}$ y se considera hipertensión cuando es mayor de $10 \mathrm{~mm} \mathrm{Hg}$. En este artículo, se describe el caso de una paciente de 5 años con un cuadro de hipertensión portal secundario a várices esofágicas y trombosis de la vena porta, confirmado por endoscopia de vías digestivas alta y angioresonancia magnética. La paciente fue atendida en la Fundación Clínica Infantil Club Noel de la ciudad de Cali, Colombia, entre los meses de diciembre del 2018 y febrero del 2019.

Palabras claves: hipertensión portal, várices esofágicas, trombosis de la vena porta

\section{Secondary portal hypertension to portal venous thrombosis and esophageal varices}

\begin{abstract}
Portal hypertension is defined as the pathological increase in the portal pressure gradient, which is the difference between the pressure of the portal vein and the inferior vena cava. Normally portal vein pressure ranges between $1-5 \mathrm{mmHg}$ and is considered hypertension when it is higher than $10 \mathrm{mmHg}$.

In this study the case of a 5-year-old patient that suffers from secondary portal hypertension to portal venous thrombosis and esophageal varices is presented. The diagnostic is confirmed by an endoscopy of the upper gastrointestinal tract and by a magnetic angioresonance. The patient was treated at the Fundacion Clinica Infantil Club Noel located in Cali, Colombia, between the months of December 2018 and February 2019.
\end{abstract}

Keywords: portal hypertension, esophageal varices, portal vein thrombosis

\section{Introducción}

La hipertensión portal se define como el aumento patológico del gradiente de presión portal que es la diferencia entre la presión de la vena porta y la vena cava inferior, el valor normal es entre 1-5 $\mathrm{mm} \mathrm{Hg}$ y se considera hipertensión cuando es mayor de $10 \mathrm{~mm} \mathrm{Hg}$; en un $60 \%$ de los pacientes descompensados hay presencia de várices esofágicas (1) .

Las principales causas de hipertensión portal son: la trombosis portal, cirrosis hepática, atresia biliar, colestasis intrahepática progresiva familiar, colangitis esclerosante primaria, hepatitis autoinmune, hepatitis $\mathrm{B}$ y $\mathrm{C}$ crónica, deficiencia de alfa-antitripsina, fibrosis hepática congénita, hepatoblastoma, carcinoma hepatocelular, idiopático, síndrome de Budd-chiari, falla cardíaca congestiva y obstrucción de la vena cava inferior (2) .

La trombosis portal es la causa del $54 \%$ de los casos de hipertensión portal en pediatría y del $68-84 \%$ de las hemorragias de vías digestivas altas en pediatría (3) hasta el 70\% de los pacientes con trombosis de la vena porta tienen várices esofágicas y su riesgo de sangrado es hasta del 50\% antes de los 16 años, con una mortalidad poco caracterizada del $0-2 \%(4)$. 
Se describe caso de paciente de 5 años quien presentó hipotensión portal secundario a várices esofágicas y trombosis de la vena porta confirmado por endoscopia de vías digestivas alta y angioresonancia magnética.

\section{Presentación del caso}

Paciente de 5 años con 2 días de dolor abdominal, distensión abdominal, sin otra sintomatología. Inicialmente es valorada en periferia donde encuentran esplenomegalia y trombocitopenia por lo que remiten. Al ingreso a la unidad especializada, presenta esplenomegalia de aproximadamente $5 \mathrm{~cm}$ por debajo del reborde costal y $9 \mathrm{~cm}$ de la línea media.

Toman paraclínicos que muestran pancitopenia (leucocitos 3.77 , hb 10.7 y plts 55000), transaminasas y bilirrubinas normales, ecografía de abdomen muestra hepatoesplenomegalia, pericolecistitis, ectasia del colédoco, TAC de abdomen que muestra esplenomegalia $163 \mathrm{~mm}$, no se define anatomía usual de la vena porta extra hepática, estructura vascular prominente a la altura del hilio hepático que se extiende por el flanco derecho en dirección caudal aparentemente continua con la arteria mesentérica superior.

Se realizan pruebas reumatológicas: ANAS 1:60, Anticuerpo Anti-DNA 1/10, SM 5.3, SA RO 3.31 LA 3.68 , inmunoglobulinas normales y C3 118 Y C4 20 y un aspirado de medula ósea negativo para enfermedad linfoproliferativa.

Es valorada por gastroenterología pediátrica quien considera descartar hipertensión porta, con endoscopia de vías digestivas alta donde se encuentran várices esofágicas grado II y várices en techo gástrico. Además, se practicó angioresonancia de abdomen donde se evidenció trombosis de la vena porta principal en relación a transformación cavernomatosa con reconstitución de las venas porta intrahepática derecha e izquierda y sus colaterales. Se inician manejo con propanolol $1 \mathrm{mg} / \mathrm{kg} /$ día, omeprazol $1 \mathrm{mg} / \mathrm{kg} /$ día y Enoxaparina $1 \mathrm{mg} / \mathrm{kg} /$ dosis cada 12 horas, además de esto, se ordenan pruebas para descartar trastornos de la coagulación. Encontrando un factor V de Leiden bajo (39), durante la hospitalización continúa con trombocitopenia en niveles que oscilan entre 55000-47000 requiriendo transfusiones, además de aumento progresivo de transaminasas y tiempos de coagulación.

Finalmente, la paciente es llevada a junta médica en donde se define que requiere de valoración y manejo integral por parte de hepatología pediátrica para realización de posible derivación tipo shunt y endoscopias de vías digestivas alta + ligadura y escleroterapia de várices esofágicas, por lo que es remitida a institución de IV nivel para realización de procedimiento.

\section{Discusión}

La trombosis de la vena porta es una de las principales causas de hipertensión portal. En la mayoría de los casos, puede tener un curso asintomático, sin embargo, también tiene un espectro clínico amplio que puede ir desde dolor abdominal, trombocitopenia, esplenomegalia, ascitis, várices esofágicas y gástricas con hemorragia de vías digestivas cuando se produce ruptura de las mismas(5). En el caso que nos ocupa, se presentó dolor abdominal, trombocitopenia, esplenomegalia y várices esofágicas y gástricas.

En un estudio observacional realizado en Brasil entre 1990-2010 las principales manifestaciones clínicas fueron la hemorragia digestiva y la esplenomegalia(6) . Mientras que en un caso reportado en el 2001 en España , se encontró como principal síntoma esplenomegalia(7).

El diagnóstico se realiza mediante ecografía doppler abdominal, tomografía computarizada o resonancia magnética, esta última ayuda a establecer la extensión(6). En el caso presentado, se realizó ecografía de abdomen, TAC de abdomen, endoscopia de vías digestivas altas y angioresonancia de abdomen para realizar el diagnóstico, mientras que en el caso reportado en España, se realizó ecografía doppler abdominal y una endoscopia de vías digestivas(7) .

El tratamiento se basa en terapia anticoagulante con intención de recanalización parcial o completa del tronco venoso portal o de una de sus ramas, generalmente se inicia con heparinas no fraccionadas o heparinas de bajo peso molecular. Además, está indicado el inicio de beta bloqueador como el propanolol para generar vasoconstricción esplacnica con reducción del flujo portal y de la presión de las várices y así prevenir el sangrado(8).

Quirúrgicamente, se puede realizar tratamiento endoscópico con ligadura con banda o escleroterapia, de igual forma para las várices esofágicas. En cuanto a la trombosis venosa, se debe considerar la cirugía tipo shunt en donde se realiza una derivación mesentérica a porta izquierda intrahepática con un injerto yugular(5). En el caso que presentamos en este artículo, se aplicaron estas tres medidas de manera satisfactoria.

En el estudio realizado en Brasil entre 1990-2010 realizaron tratamiento con ligaduras de banda y por escleroterapia, algunos pacientes necesitaron realización de shunt (6) .En el caso reportado en España, se trató con propanolol y protectores gástricos(7).

\section{Conclusión}

La hipertensión portal es poco común en pediatría por lo que se debe conocer sus principales manifestaciones clínicas con el fin de tener una sospecha clínica y 
ordenar las ayudas diagnósticas pertinentes para inicio de tratamiento de forma precoz, con el fin de evitar presencia de hemorragias en las vías digestivas alta.

\section{Reconocimientos y agradecimientos}

Agradecemos a la Fundación Clínica infantil Club Noel y a la Universidad Libre Seccional Cali.

\section{Referencias}

1. Hadi M., Mohsen S., Khoshkhui M., Malekpour A., Etiology of portal hypertension in children: a single center's experiences. Middle East Journal of Digestive Diseases. 2012;4 (4): 206-2010

2. Barbon C. Pediatric portal hypertension. Nurse Pract 2017;45(5); p. 35-42 doi: 10.1097/01. NPR.0000515427.91649.91

3. Feldman A., Sokol R. Non cirrhotic Portal Hypertension in the pediatric population. Clinical liver disease 2015; 5(5); p. 116-119 doi: https:// doi.org/10.1002/cld.471

4. Ling S., Portal hypertension in children. Clinical liver disease 2012;1(5); p. 139-142 doi: https:// doi.org/10.1002/cld.79

5. Camarera C. Hipertensión portal. Protocolos diagnostico-terapéutico de gastroenterología, hepatología y nutrición pediátrica SEGHNPAEP. Sociedad Española de pediatría. 2010. 2 edición: p. 221-226.

6. Menezes P., Rodrigues A., Druve E., Shinfay M.m Leitao M., Penna JF. Portal vein trombosis in children and adolescents: 20 years experiencia of a pediatric hepatology reference center. Arq Gastroenterol. 2012;49(1): p. 69-76 Doi: http:// dx.doi.org/10.1590/S0004-28032012000100012

7. Senderos B., Garcia MC., Casanova A. Trombosis de la vena porta. An Esp Pediatr 2001.55.(6); p. 565-568. Doi: 10.1016/S16954033(01)77740-5

8. Seijo S., Garcia JC. Trombosis portal. Gastroenterol Hepatol 2010.33 (3); p. 179-190 Doi: 10.1016/j.gastrohep.2009.04.002 